\title{
WEIGHTED INTEGRAL INEQUALITY AND APPLICATION IN UNIFORM STABILITY FOR A NONLINEAR SYSTEM WITH MEMORY
}

\author{
KAIQIANG LI AND FUSHAN LI*
}

Abstract. In this paper, we consider the viscoelastic system

$$
\boldsymbol{u}_{t t}-\mu \Delta \boldsymbol{u}-(\lambda+\mu) \nabla(\operatorname{div} \boldsymbol{u})+\int_{0}^{t} g(t-s) \operatorname{div}[a(x) \nabla \boldsymbol{u}(s)] d s+b(x) \boldsymbol{h}\left(\boldsymbol{u}_{t}\right)=\boldsymbol{f}(\boldsymbol{u})
$$

with initial conditions and boundary conditions. Under some assumptions on the relaxation function $g$, and other functions $\boldsymbol{h}$ and $\boldsymbol{f}$, without constructing any auxiliary functional, by establishing weighted integral inequality on the energy functional, we obtain a general energy decay formula for the solution, such that the usual exponential decay results and the polynomial decay results are only special cases, respectively.

Mathematics subject classification (2010): 35L05, 35L15, 35L70.

Keywords and phrases: Viscoelastic system, relaxation function, control functions, asymptotic behavior.

\section{REFERENCES}

[1] F. Alabau-Boussouira, P. Cannars a, D. Sforza, Decay estimates for second order evolution euations with memory, Journal of Functional Analysis. 254 (5) (2008), 1342-1372.

[2] M. M. Cavalcanti, H. P. OQuendo, Frictional versus viscoelastic damping in a semi-linear wave equation, SIAM J. Control Optim. 42 (4) (2003), 1310-1324.

[3] L. C. Evans, Partial Differential Equations (2nd ed.), in: Grad. Stud. Math., vol. 19, Amer. Math. Soc., Providence, RI, 2010.

[4] H. D. Fernández Sare, J. E. Muñoz Rivera, Optimal rates of decay in 2-d thermoelasticity with second sound, J. Math. Phys. 53(2012), 073509.

[5] Y. HU, Global solvability in thermoelasticity with second sound on the semi-axis, J. Partial Differ. Eqns. 25 (2012), 37-68.

[6] K. Jina, J. LiAng, T. XIAO, Coupled second order evolution equations with fading memory: Optimal energy decay rate, J. Differential Equations 257 (2014), 1501-1528.

[7] F. LI, Global existence and uniqueness of weak solution for nonlinear viscoelastic full Marguerre-von Karman shallow shell equations, Acta Mathematica Sinica, English Series 25 (12) (2009), 2133-2156.

[8] F. LI, Limit behavior of the solution to nonlinear viscoelastic Marguerre-von Kármán shallow shells system, J. Differential Equations 249 (2010), 1241-1257.

[9] F. LI, Y. BAI, Uniform rates of decay for nonlinear viscoelastic Marguerre-von Kármán shallow shell system, J. Math. Anal. Appl. 351 (2009), 522-535.

[10] F. LI, Y. BAO, Uniform stability of the solution for a memory-type elasticity system with nonhomogeneous boundary control condition, J. Dyn. Control. Syst. 23 (2017), 301-315.

[11] F. LI, G. DU, General energy decay for a degenerate viscoelastic Petrovsky-type plate equation with boundary feedback, J. Appl. Anal. Comput. 8 (1) (2018), 390-401.

[12] F. LI, C. ZHAO, Uniform energy decay rates for nonlinear viscoelastic wave equation with nonlocal boundary damping, Nonlinear Anal. 74 (2011), 3468-3477.

[13] F. LI, Z. ZHAO, Y. CHEN, Global existence uniqueness and decay estimates for nonlinear viscoelastic wave equation with boundary dissipation, Nonlinear Analysis: Real World Applications 12 (2011), 1770-1784. 
[14] P. MarTinez, A new method to obtain decay rate estimates for dissipative systems with localized damping, ESAIM Contr. Optim. CA. 4 (1999), 419-444.

[15] M. I. Mustafa, Boundary stabilization of memory-type thermoelasticity with second sound, Z. Angew. Math. Phys. 63 (2012), 777-792.

[16] J. Y. PARK, S. H. PARK BUSAN, Existence and asymptotic stability for viscoelastic problems with nonlocal boundary dissipation, Czechoslovak Math. J. 56 (131) (2006), 273-286. 